\title{
Electrophotocatalysis: Cyclic Voltammetry as an Analytical Tool
}

\author{
Cyrille Costentin* ${ }^{a, b}$, Jérôme Fortage ${ }^{a}$, Marie-Noëlle Collomb ${ }^{a}$
}

${ }^{a}$ Univ. Grenoble Alpes, CNRS, DCM, 38000 Grenoble, France. ${ }^{b}$ Université de Paris, 75013 Paris, France. Supporting Information

ABSTRACT: Electrophotocatalysis (e-PC) is currently experiencing a renewed interest. Taking advantage of the highly oxidizing or reducing power of excited state of electrogenerated ion radicals it allows to perform thermodynamically difficult redox reactions. However, e-PC is facing various specific issues, such as its fundamentally heterogeneous nature implying that mass transport is coupled to chemical reactions and light absorption; back electron transfer of the ion radical excited state with the electrode; local heating near the electrode surface modifying mass transport conditions. Herein we address these issues in the context of cyclic voltammetry as an analytical tool and we provide a rational framework for kinetic studies of electrophotocatalytic reactions under realistic conditions and hypothesis based on literature data. This approach may be beneficial to rationalize the design and the efficiency of present and future e-PC systems.

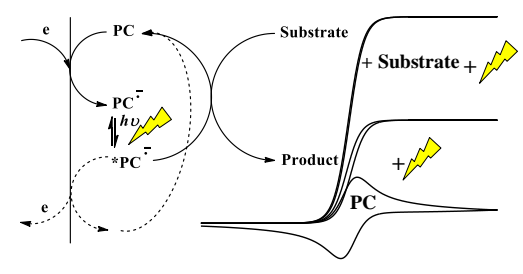

\section{Introduction}

Harnessing the energy of light to perform redox reactions is a very active domain referred to as photoredox catalysis. ${ }^{1,2}$ In its most common form, molecular photocatalysis of redox reactions is a process where a photocatalyst (PC) is excited and triggers an electron transfer reaction either via oxidative or reductive quenching by a sacrificial electron acceptor (SA) or a sacrificial electron donor (SD). This process takes advantage of the fact that the excited state of the photocatalyst $* \mathrm{PC}$ is a stronger oxidant and a stronger reductant than its ground state. The anion or cation radical of the photocatalyst $\left(\mathrm{PC}^{*-}\right.$ or $\left.\mathrm{PC}^{\circ+}\right)$ thus formed is then either oxidized or reduced by a substrate $\left(\mathrm{S}_{\mathrm{ox}}\right.$ or $\mathrm{S}_{\mathrm{red}}$ ) to regenerate PC (Scheme 1). A molecular catalyst can be additionally used to pick up the hole from the photoinduced oxidant $\left(\mathrm{PC}^{\circ+}\right)$ or the electron from the photoinduced reductant $\left(\mathrm{PC}^{*}\right)$ ' and activate the substrate $\left(\mathrm{S}_{\mathrm{ox}}\right.$ or $\left.\mathrm{S}_{\mathrm{red}}\right)$. Similarly, a molecular catalyst can mediate the electron transfer from *PC to SA or SD. ${ }^{2,3,4,5,6,7,8,9}$ The sacrificial acceptor or donor can also be replaced by a semiconductor electrode (injection of electrons in the conduction band or injection of holes in the valence band) as in dye-sensitized solar cells (DSCC). ${ }^{10}$, Both light absorption and charge separation can be directly combined in a single step using a semiconductor electrode in which a hole and an electron are generated upon light absorption. Then the hole (photoanode) or the electron (photocathode) reacts at the surface of the semiconductor with the substrate. Such an interfacial photoelectrochemistry (iPEC) process is known as "photoelectrocatalysis". ${ }^{11}$ It is demanding as a single material (the semiconductor electrode) has to be engineered to perform efficiently two very different functions: light absorption to separate charges and surface reactivity with the substrate. It is therefore the reason why a buried junction con- figuration is usually preferred in the context of artificial photosynthesis. ${ }^{12}$

Scheme 1. Principle of common form of molecular photoredox catalysis

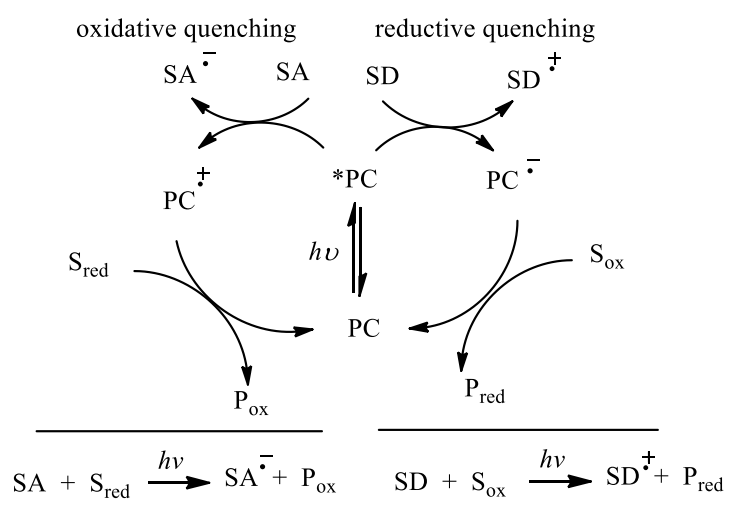

Alternatively, performing thermodynamically difficult oxidation or reduction can be achieved via the very strong oxidative or reductive power of radical or ion radical excited state as reported some time ago ${ }^{13}$ or more recently. ${ }^{14}$ Several examples of such a process can be found both for oxidation ${ }^{15}$ and reduction. ${ }^{16,17,18}$

Scheme 2. Principle of photoredox catalysis via ion radical excited states 


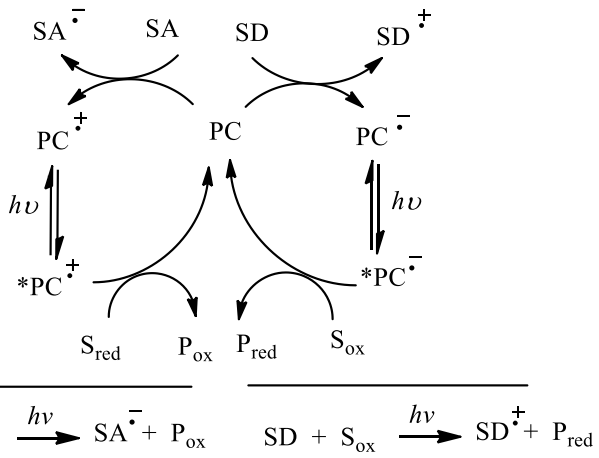

As compare to common molecular photoredox catalysis, the basic principle is to invert the order of light absorption and electron transfer to or from the sacrificial acceptor or donor so as to generate a very highly reducing or oxidative species (Scheme 2). The sacrificial electron donor or acceptor can be a homogeneous molecule ${ }^{19}$ or be conveniently replaced by an electrode. Because a complementary redox reaction is necessarily occurring at the counter electrode, the overall process has been recently proposed in a comprehensive review on synthetic photoelectrochemistry to be referred to as electrochemically mediated photoredox catalysis. ${ }^{20}$ To avoid confusion with common photoredox catalysis we prefer to use here the term Electrophotocatalysis (e-PC). The principle of e-PC is sketched in Scheme 3 in the case of a reduction (transposition to oxidation is straightforward).

Scheme 3. Principle of e-PC

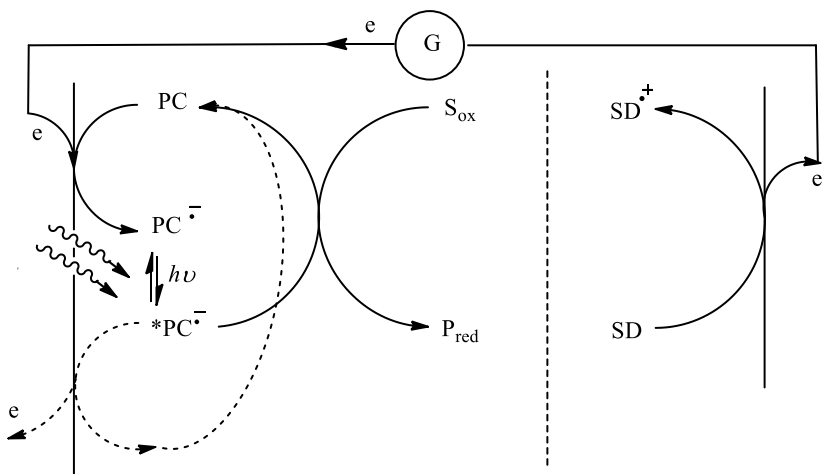

e-PC is currently attracting a renewed attention, ${ }^{21,22,23,24,25,26}$ in part from its ability to generate highly energetic chemical intermediates (e.g. benzene radical cation ${ }^{22}$ ) to form new bonds. In the case of oxidation, it allows avoiding strong oxidants, often toxic. The case of reduction is also of potential interest as scalable strong reductive reagents are not yet available. ${ }^{27}$ Some examples are given in Scheme 4.

In the following, we will focus on electro-photo-reduction as sketched in Scheme 3. The process bears some similarities with redox catalysis of electrochemical reactions considering the catalytic redox couple as $\mathrm{PC}+h v / * \mathrm{PC}^{\bullet-} .{ }^{28}$ However, the presence of light allows for generating a strong reductant at a moderate potential whereas redox catalysis does not provide more reducing power than the applied potential, catalysis arising from spreading reductants in a tri-dimensional space out of two-dimensional electrode surface.

Scheme 4. Examples of electro-photo-generated strong oxidants ${ }^{15,20,22}$ and reductants ${ }^{20,29}$
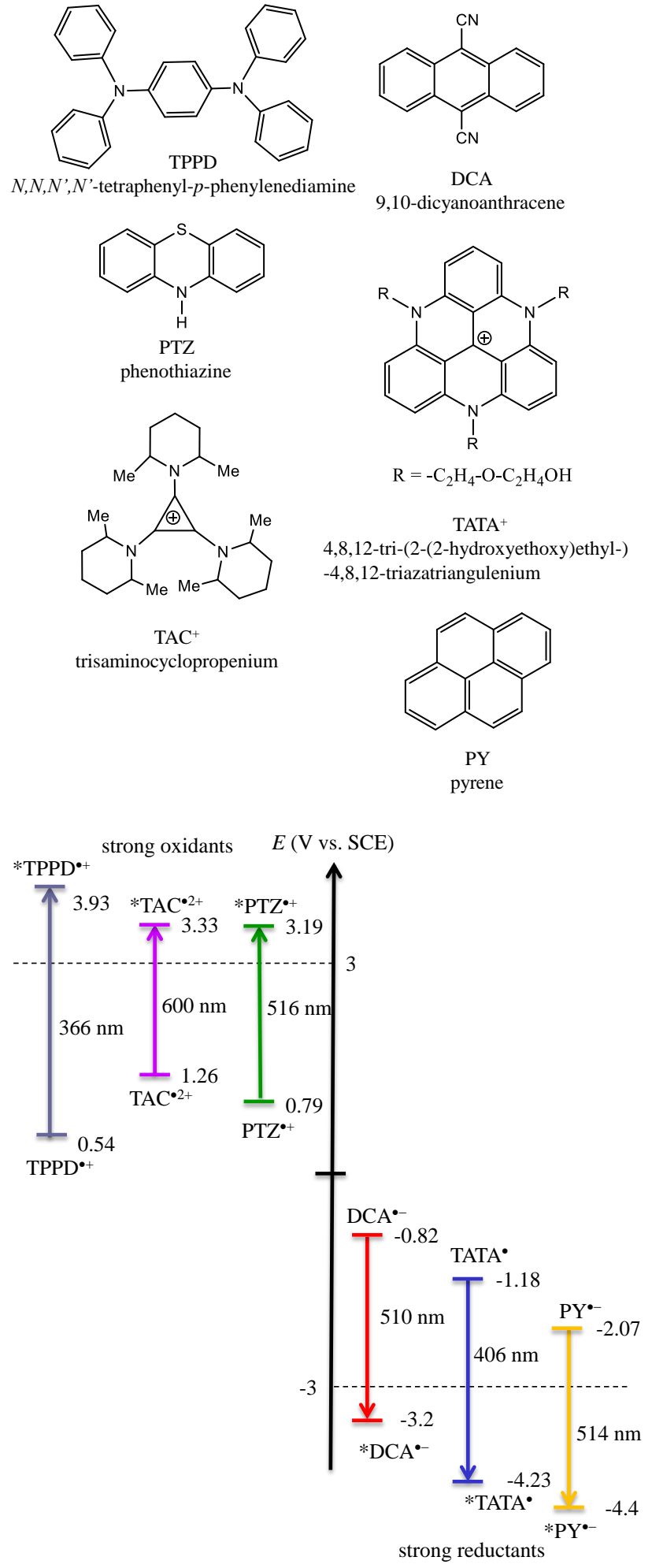

As recently pointed out, ${ }^{20}$ several challenges are faced in the endeavor to design an e-PC system. First, the photocatalyst (PC) has to undergo a facile one-electron transfer at the electrode to yield a primary radical $\left(\mathrm{PC}^{\bullet-}\right.$ in Scheme 3$)$ stable enough so that it can be excited. Second, this primary radical $\left(\mathrm{PC}^{\bullet-}\right)$ has to absorb light at a different wavelength than the initial species (PC). Third, the radical excited state $\left({ }^{*} \mathrm{PC}^{\bullet-}\right)$ has to be long-lived enough to interact with the substrate $\left(\mathrm{S}_{\mathrm{ox}}\right)$. We note that if the first condition is easily met (i.e. very stable primary radical) then a two-steps e-PC process can be designed 
(decoupled photoelectrochemistry $\mathrm{dPEC}^{20}$ ), akin to the "cation pool" method in redox catalysis, ${ }^{30}$ in which a solution of $\mathrm{PC}^{\bullet-}$ is obtained via complete electrolysis of a PC solution, then the reactant $S_{o x}$ is introduced under illumination (Scheme 5). Additionally this decoupled approach might be useful for mechanistic investigation, i.e. for example characterization of $* \mathrm{PC}^{\bullet-}$ photophysics and quenching by the substrate $S_{\mathrm{ox}}$. Performing a one step (one pot) e-PC process allows circumventing the requirement for $\mathrm{PC}^{\bullet-}$ solution to be stable and to proceed to manipulation in the course of the reaction. That been said, one has to recognize that the one step e-PC process also requires optimized conditions to reach an efficient transformation.

Scheme 5. Two-steps e-PC

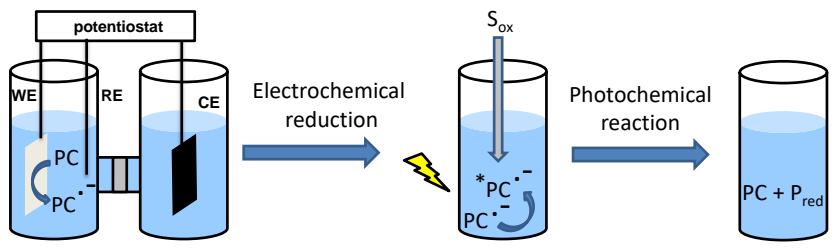

Among the various specific issues, we can mention that (i) as any electrochemically induced transformation, this process is fundamentally heterogeneous because $\mathrm{PC}^{\bullet-}$ is produced at the electrode surface and therefore mass transport is coupled to chemical reactions and light absorption; (ii) as sketched in Scheme 3 (dotted arrows) the highly reducing $* \mathrm{PC}^{\bullet-}$ is generated at a potential at which it can be immediately oxidized leading to an energy wasting back electron transfer; (iii) nonradiative deactivation of $* \mathrm{PC}^{\bullet-}$ generated in the vicinity of the electrode may also induce local heating modifying mass transport conditions (convection). Addressing these issues and more generally investigating e-PC mechanism calls for the use of analytical techniques such as advanced spectroscopic, spectroelectrochemical and computational tools. ${ }^{20} \mathrm{We}$ believe that well developed classical electrochemical techniques such as cyclic voltammetry (CV) can be relevant to explore e-PC as will be shown in the present contribution. Cyclic voltammetry was used in the very early investigation of e-PC systems, back in the 70's, ${ }^{31}$ with identification of specific challenges such as the influence of thermal convection or back electron transfer ${ }^{32}$ but without quantitative analysis. To the best of our knowledge, no fundamental kinetic studies have been performed on e-PC beside preliminary cyclic voltammetry studies 16,32 and analytical tools are clearly lacking. A formal kinetic analysis is thus now performed which provides a framework for ongoing experimental studies.

\section{Results and Discussion}

We consider a system as represented in Scheme 3 and we focus on process taking place at the working electrode (left electrode in Scheme 3) which potential is controlled by a potentiostat in a $\mathrm{CV}$ experiment. The molecular photocatalyst (PC, assumed to be a neutral species for the sake of simplicity) diffuses in solution (diffusion coefficient $D$ ) and can be reduced at the electrode surface. Upon steady-state irradiation with a photon flux $(I)$, that we assume to be from the backside of the electrode, the anion radical $\mathrm{PC}^{\bullet-}$ is the only species absorbing light (molar extinction coefficient $\varepsilon$ ) to generate the excited state ${ }^{*} \mathrm{PC}^{\bullet}$. While diffusing, the excited state fate is threefold: non-radiative deactivation (rate constant $k_{b}=1 / \tau_{b}$ in $\mathrm{s}^{-1}$ where $\tau_{b}$ is the ${ }^{*} \mathrm{PC}^{\bullet-}$ lifetime); reaction with a substrate $\left(\mathrm{S}_{\mathrm{ox}}\right)$ regenerating $\mathrm{PC}$ and re-oxidation at the electrode surface also regenerating $\mathrm{PC}$. The latter corresponds to back electron transfer because $* \mathrm{PC}^{\bullet-}$ is a strong reductant and eager to transfer an electron to the electrode in the range of potential corresponding to the $\mathrm{CV}$ scan. Back electron transfer can also occur via the homogeneous irreversible reaction:

$* \mathrm{PC}^{-}+\mathrm{PC} \longrightarrow \mathrm{PC}+\mathrm{PC}^{-}$

However, we note that this downhill bimolecular reaction can be considered as limited by diffusion $\left(k_{d i f} \approx 10^{10} \mathrm{M}^{-1} \mathrm{~s}^{-1}\right)$ and, hence, considering a typical $\mathrm{mM}$ concentration of $\mathrm{PC}$, its contribution to ${ }^{*} \mathrm{PC}^{\bullet-}$ is negligible compared to the non-radiative deactivation: $k_{d i f}[\mathrm{PC}] \approx 10^{7} \mathrm{~s}^{-1}<k_{b}=1 / \tau_{b}$ taking into account that the lifetimes of photocatalyst anion radical excited states are typically sub-nanosecond. ${ }^{33,34,35}$ Therefore, in the following, we will discard this homogeneous reaction.

In a cyclic voltammetry experiment at a planar microelectrode which dimension (typical disk electrode diameter $0.3 \mathrm{~cm}$ ) is greater than the diffusion layer thickness, diffusion can be assumed to be linear. The diffusion layer thickness is indeed $\sqrt{D R T / F v}$ of the order of $10^{-3} \mathrm{~cm}$ taking $D=10^{5} \mathrm{~cm}^{2} \mathrm{~s}^{-1} ; T$ $=298 \mathrm{~K}$ and $v=0.1 \mathrm{~V} \mathrm{~s}^{-1}$ ( $F$ is the Faraday and $R$ the gas constant). Provided the scan rate is not too slow (> $\left.20 \mathrm{mV} \mathrm{s}^{-1}\right)$, linear diffusion can be safely assumed to be semi-infinite, i.e. not perturbed by natural convection. This state of affair is not necessarily true anymore upon irradiation as it causes an increase of the temperature in the immediate vicinity of the electrode thus boosting convection. ${ }^{32}$ Consequently, we consider a planar non-infinite diffusion with a maximal diffusion layer thickness equal to $\delta$ being a function of the photon flux $I$ passing through the diffusion-reaction layer. We will show later on how $\delta$ can be experimentally evaluated from $\mathrm{CV}$. The maximal diffusion layer size $\delta$ defines the maximal space zone in the solution in which the photocatalytic process can take place because the concentration of $\mathrm{PC}^{\circ-}$ is nil beyond this layer.

Considering an irradiation of the solution from the backside of the electrode (using for example a transparent ITO electrode), light is absorbed by the electrogenerated anion radical $\mathrm{PC}^{\bullet-}$ and therefore the photon flux I may decrease within the diffusion-reaction layer. However, considering a maximal concentration of $\mathrm{PC}^{\bullet-}$ of $1 \mathrm{mM}$, a typical molar extinction coefficient $\varepsilon \approx 10^{4} \mathrm{M}^{-1} \mathrm{~cm}^{-136}$ and a diffusion layer length of $310^{-3} \mathrm{~cm}$, then the photon flux attenuation is $I / I_{0} \approx 0.93$ ( $I_{0}$ is the photon flux at the electrode surface). We can thus conclude that the light penetration length is much larger than the diffusion layer size and therefore consider a uniform photon flux ( $I=I_{0}$ ) throughout the diffusion-reaction layer. Moreover, this observation implies that the mode of irradiation of the electrochemical cell (from the backside of the working electrode or from other directions) does not matter as light is only absorbed in a diffusion-reaction layer much thinner than the light penetration length.

Within the above framework (constant and uniform photon flux $I$ and maximal diffusion layer size $\delta$ ), we will as a first task described the $\mathrm{CV}$ electrophotoreduction behavior of PC in the absence of substrate. Then, we will describe the CV e-PC 
response in the presence of the substrate $S_{o x}$.

The photocatalyst reduction is characterized thermodynamically by a standard potential $E_{\mathrm{PC}}^{0}$ and kinetically by a standard rate constant $k_{S}$ and a transfer coefficient $\alpha$ assumed to be 0.5 . The light absorption pseudo-first order process has a rate constant $\varepsilon I$. Non radiative deactivation of the excited state $* \mathrm{PC}^{\bullet-}$ occurs with a rate constant $k_{b}$ and $* \mathrm{PC}^{\bullet-}$ is oxidized at the electrode surface at a very high rate because the corresponding standard potential $E_{2}^{0}$ is much more negative than $E_{\mathrm{PC}}^{0}$. Therefore the concentration of $* \mathrm{PC}^{\bullet-}$ at the electrode surface is zero. Scheme 6 summarized this electrophotoreduction process. For the sake of simplicity, we assume that $\mathrm{PC}^{\bullet}$ cannot be further reduced so that there is no homogeneous reduction of $\mathrm{PC}^{\bullet-}$ by $* \mathrm{PC}^{\bullet-}$ producing $\mathrm{PC}^{2-}$ as might be the case with some photocatalysts, e.g. pyrene. ${ }^{32}$

Scheme 6. Electrophotoreduction of the photocatalyst PC

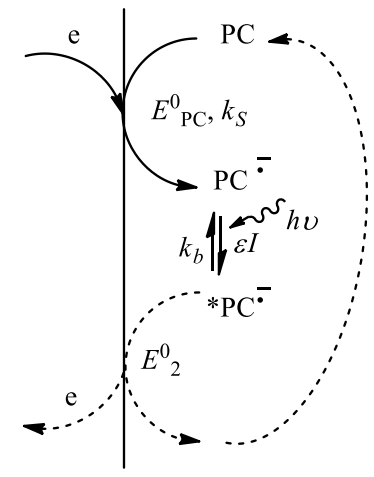

Considering diffusion reactions equations for all three species $\mathrm{PC}, \mathrm{PC}^{\bullet-}$ and $* \mathrm{PC}^{\bullet-}$ together with initial and boundary conditions associated with a CV experiment, it can be shown (see Supporting Information (SI)), that the system is governed by the following four dimensionless parameters: $K=\frac{\varepsilon I}{k_{b}}$; $\lambda_{b}=\frac{k_{b}}{F v / R T}=\frac{\tau_{C V}}{\tau_{b}} ; \Lambda=\frac{k_{S}}{\sqrt{D F v / R T}}$ and $l=\frac{\delta}{\sqrt{D R T / F v}}$.

The first parameter is the apparent equilibrium constant for $\mathrm{PC}^{\bullet-}$ light absorption/ $* \mathrm{PC}^{\bullet-}$ deactivation. For systems of interest, we have $K=\frac{\varepsilon I}{k_{b}} \ll 1$. Indeed, the lifetime of anion radical excited states is nano or sub-nanosecond, hence, $k_{b}>10^{9} \mathrm{~s}^{-1}$ whereas $\varepsilon \approx 10^{7} \mathrm{~mol}^{-1} \mathrm{~cm}^{2} 34$ and a typical value for the photon flux is $I \approx 2 \times 10^{-7} \mathrm{~mol} \mathrm{~s}^{-1} \mathrm{~cm}^{-2} 37$ leading to $\varepsilon I \approx 2 \mathrm{~s}^{-1}<<k_{b}$. As a consequence the concentration of $* \mathrm{PC}^{\bullet-}$ does not build-up in the diffusion-reaction layer. The second parameter is a comparison of the excited state anion radical lifetime and the characteristic time of the $\mathrm{CV}$, $\tau_{C V}=R T / F v$. For typical scan rate values, $\lambda_{b}=\tau_{C V} / \tau_{b}$ $\gg 1$ indicating that the apparent equilibrium between $\mathrm{PC}^{\bullet-}$ and $* \mathrm{PC}^{\bullet-}$ is met. The third parameter $\Lambda$ characterizes the kinetic of the interfacial electron transfer compared to the rate of diffusion. Finally, $l$ indicates on whether diffusion is semi- infinite $(l>>1)$ or constraint within a layer of thickness $\delta$ ( $l$ $<1)$.

The condition $\lambda_{b}=\tau_{C V} / \tau_{b}>1$ allows for considering pure kinetics conditions, i.e. a steady state approximation for the quantity $\left(K\left[\mathrm{PC}^{\bullet-}\right]-\left[\mathrm{PC}^{\bullet-*}\right]\right)$ due to compensation of diffusion and reaction (see SI). As a consequence and taking into account that $K \ll<1$, it is shown that the system is governed by only three parameters: $l, \Lambda$ and $K \sqrt{\lambda_{b}}$, and that a closed-form of the $\mathrm{CV}$ dimensionless equation can be derived:

$\int_{0}^{\tau} \psi \times \Theta^{-1}\left[\frac{\tanh (l \sqrt{s})}{\sqrt{s}}\right]_{\tau-\eta} d \eta=\frac{1-\psi \frac{\exp (-\xi / 2)}{\Lambda}}{1+\exp (-\xi)+\exp (-\xi / 2) \frac{K \sqrt{\lambda_{b}}}{\Lambda}}$

with the dimensionless current $\psi=\frac{i}{F S C_{\mathrm{PC}}^{0} \sqrt{D F v / R T}}\left(C_{\mathrm{PC}}^{0}\right.$

is the bulk concentration of PC, $S$ the electrode surface area),

the dimensionless potential $\xi=-\frac{F}{R T}\left(E-E_{\mathrm{PC}}^{0}\right) \quad$ and $\Theta^{-1}[f(s)]_{\tau-\eta}$ representing the inverse Laplace transform of function $f(s)$ at value $\tau-\eta$ where $\tau=t / \tau_{C V}$.

In the absence of irradiation (dark behavior), $K \sqrt{\lambda_{b}}=0$ and $l>>1$ so that the $\mathrm{CV}$ equation becomes:

$\psi \frac{\exp (-\xi / 2)}{\Lambda}+\frac{1+\exp (-\xi)}{\sqrt{\pi}} \int_{0}^{\tau} \frac{\psi}{\sqrt{\tau-\eta}} d \eta=1$

which, with no surprise, is the exact CV expression of a chemically reversible one electron redox coupled with possible interference of electron transfer kinetics depending on the value of $\Lambda .{ }^{38}$ Characteristics of the electron transfer kinetics ( $k_{S}$ ) as well as the diffusion coefficient $(D)$ and the standard potential ( $E_{\mathrm{PC}}^{0}$ ) can then easily be extracted from peak potentials and intensity from $\mathrm{CV}$ recorded at various scan rates.

Under irradiation, if electron transfer kinetics is fast $(\Lambda \rightarrow \infty)$, then the $\mathrm{CV}$ equation simplifies to:

$\int_{0}^{\tau} \psi \times \Theta^{-1}\left[\frac{\tanh (l \sqrt{s})}{\sqrt{s}}\right]_{\tau-\eta} d \eta=\frac{1}{1+\exp (-\xi)+\exp (-\xi / 2) \frac{K \sqrt{\lambda_{b}}}{\Lambda}}$

and the system is controlled by two parameters $l=\frac{\delta(I)}{\sqrt{D R T / F v}}$ and $\frac{K \sqrt{\lambda_{b}}}{\Lambda}=\frac{\varepsilon I \sqrt{D / k_{b}}}{k_{S}}$ and a zone diagram can be drawn representing the various cases that can be obtained depending on the values of the governing parameters and how manipulation of the operational parameters (scan rate and light intensity) or intrinsic properties $\left(k_{S}, \varepsilon\right.$ or $\left.k_{b}\right)$ can move the system from one situation to another one (figure 1). For the sake of simplicity, the arrow for $I$ in the compass of the zone diagram was drawn assuming that the maximal diffusion layer thickness $\delta$ is proportional to $1 / I$. 
$\log \left(\frac{\delta}{\sqrt{D R T / F v}}\right)$

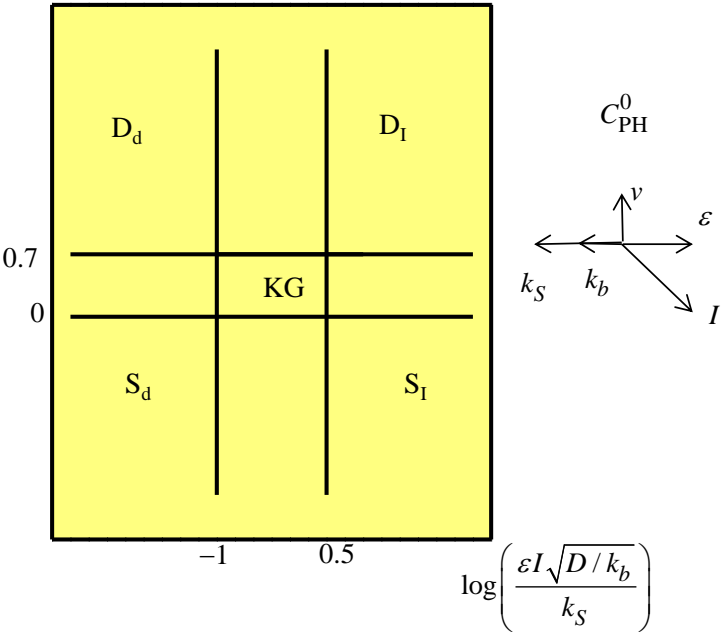

Figure 1. Zone diagram for electrophotoreduction $\mathrm{CVs}$ with $K<<1$ and $\Lambda \rightarrow \infty$ and pure kinetics conditions $\lambda_{b} \gg 1$. Dimensionless expressions and characteristics of the limiting CVs corresponding to zones $D_{d}, S_{d}, D_{I}$ and $S_{I}$ are given in Table 1. (zones D correspond to a semi-infinite diffusion type wave; zones $\mathrm{S}$ correspond to sigmoid type CVs due to limited diffusion layer thickness). KG corresponds to the general case described by the complete $\mathrm{CV}$ equation given in the text. Zones without names correspond to transition zones. The compass rose summarized how the variations of the parameters move the system from one zone to the other.

When $\frac{K \sqrt{\lambda_{b}}}{\Lambda}=\frac{\varepsilon I \sqrt{D / k_{b}}}{k_{S}}<<1$ (left side of the zone diagram), i.e. fast interfacial electron transfer compared to dynamics of light absorption, the photochemical process has no effect on the CV and irradiation only induces local heating and increase of convection. This effect tends to transform the peak shaped $\mathrm{CV}$ into a stationary sigmoidal $\mathrm{CV}$ as shown on figure 2a.
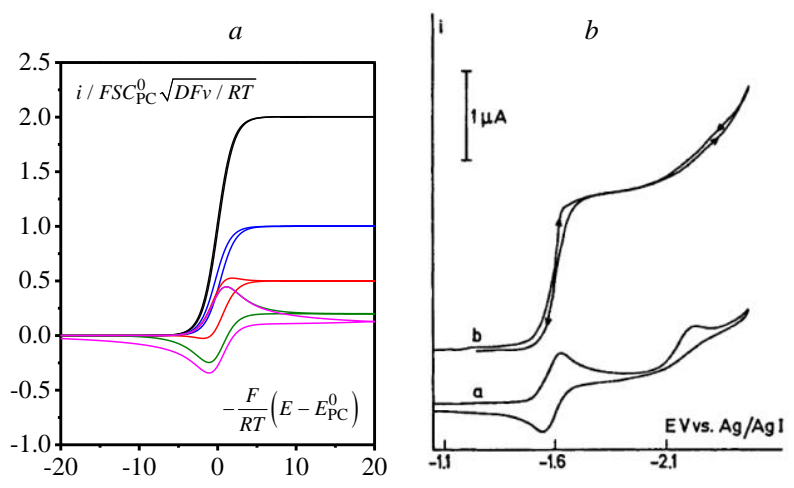

Figure 2. (a) Simulations of $\mathrm{CVs}$ in the limiting case $\frac{K \sqrt{\lambda_{b}}}{\Lambda}<<1$ as function of $l=\frac{\delta}{\sqrt{D R T / F v}}=0.5$ (black), 1 (blue), 2 (red), 5 (green), 10 (magenta). (b) CVs of pyrene (5 $\mathrm{mM}$ ) in DMF + 0.1 M tetrabutylammonium iodide a: in the dark, b: under irradiation $(514.5 \mathrm{~nm}), v=0.04 \mathrm{~V} \mathrm{~s}^{-1}$. Repro- duced from figure 1 in reference 31 .

The corresponding plateau current allows for an easy evaluation of the diffusion layer maximal thickness $\delta$ ( $\left.i_{p l}=F S D C_{\mathrm{PC}}^{0} / \delta\right)$ and hence to a calibration of the $\delta=f(I)$ experimental relationship. Figure $2 \mathrm{~b}$ reports data from reference 31 showing from the plateau current enhancement upon irradiation that $\delta=6.3 \mu \mathrm{m}(l \approx 0.8)$ taking into account an evaluation of the diffusion coefficient $D=10^{-6} \mathrm{~cm}^{2} \mathrm{~s}^{-1}$ from the peak current of the one electron reversible wave in the dark $\left(i_{p}=0.446 F S C_{\mathrm{PC}}^{0} \sqrt{D F v / R T}\right.$ with a disk electrode of 0.25 $\mathrm{mm}$ diameter).

Table 1. Dimensionless equations and characteristics of the limiting CVs of the zone diagram in figure 1.

\begin{tabular}{|c|c|c|}
\hline $\begin{array}{l}\text { Zon } \\
\mathrm{e}\end{array}$ & Equation & Characteristics $^{a}$ \\
\hline $\mathrm{D}_{\mathrm{d}}$ & $\frac{1}{\sqrt{\pi}} \int_{0}^{\tau} \frac{\psi}{\sqrt{\tau-\eta}} d \eta=\frac{1}{1+\exp (-\xi)}$ & $\begin{array}{l}i_{p}=0.446 F S C_{\mathrm{PC}}^{0} \sqrt{\frac{D F v}{R T}} \\
E_{1 / 2}=E_{\mathrm{PC}}^{0}\end{array}$ \\
\hline$S_{d}$ & $\psi \times l=\frac{1}{1+\exp (-\xi)}$ & $\begin{array}{l}i_{p l}=F S D C_{\mathrm{PC}}^{0} / \delta \\
E_{1 / 2}=E_{\mathrm{PC}}^{0}\end{array}$ \\
\hline $\mathrm{D}_{\mathrm{I}}$ & $\frac{\frac{1}{\sqrt{\pi}} \int_{0}^{\tau} \frac{\psi}{\sqrt{\tau-\eta}} d \eta=}{1+\exp \left(-\frac{\xi}{2}+\ln \frac{K \sqrt{\lambda_{b}}}{\Lambda}\right)}$ & $\begin{array}{l}i_{p}=0.446 F S C_{\mathrm{PC}}^{0} \sqrt{\alpha \frac{D F v}{R T}} \\
E_{1 / 2}=E_{\mathrm{PC}}^{0}-\frac{R T}{\alpha F} \ln \left(\frac{\varepsilon I}{k_{S}} \sqrt{\frac{D}{k_{b}}}\right.\end{array}$ \\
\hline $\mathrm{S}_{\mathrm{I}}$ & $\begin{array}{l}\psi \times l= \\
\left.\frac{1}{1+\exp \left(-\frac{\xi}{2}+\ln \frac{K \sqrt{\lambda_{b}}}{\Lambda}\right.}\right)\end{array}$ & $\begin{array}{l}i_{p l}=F S C_{\mathrm{PC}}^{0} / \delta \\
E_{1 / 2}=E_{\mathrm{PC}}^{0}-\frac{R T}{\alpha F} \ln \left(\frac{\varepsilon I}{k_{S}} \sqrt{\frac{D}{k_{b}}}\right)\end{array}$ \\
\hline
\end{tabular}

${ }^{a} E_{1 / 2}$ is the mid-point potential ( $\mathrm{D}_{\mathrm{d}}$ and $\mathrm{D}_{\mathrm{I}}$ zones) or the half-wave potential ( $\mathrm{S}_{\mathrm{d}}$ and $\mathrm{S}_{\mathrm{I}}$ zones)

When $\frac{K \sqrt{\lambda_{b}}}{\Lambda}=\frac{\varepsilon I \sqrt{D / k_{b}}}{k_{S}}>1$ (right side of the zone diagram), the photochemical process manifests itself via a shift of the CV (figure 3) with an apparent standard potential (when $l \rightarrow \infty$ ) or half-wave potential (when $l \rightarrow 0$ ) equal to:

$E_{a p}^{0}=E_{1 / 2}=E_{\mathrm{PC}}^{0}-\frac{2 R T}{F} \ln \left(\frac{\varepsilon I \sqrt{D / k_{b}}}{k_{S}}\right)$

$\frac{\varepsilon I \sqrt{D / k_{b}}}{k_{S}}$ actually measures the competition between the ability to transfer electron to the solution species (via $k_{S}$ ) and the ability to generate excited electrons within a thin layer close to the electrode (of size $\sqrt{D / k_{b}}$ ) able to be transferred 
back to the electrode. The condition $\frac{\varepsilon I \sqrt{D / k_{b}}}{k_{S}}>>1$ indicates that the latter is greater than the former and hence in a range of potential, where interfacial electron transfer rate is lower than $\varepsilon I \sqrt{D / k_{b}}$, there is no net current. Therefore, the wave is shifted to more cathodic potentials where electron transfer rate constant is larger. However, we note that such a situation ( $\left.K \sqrt{\lambda_{b}} / \Lambda>>1\right)$ is difficult to be met with most systems. Indeed, considering typical values, $\varepsilon \approx 10^{7} \mathrm{~mol}^{-1} \mathrm{~cm}^{2}$, $I \approx 2 \times 10^{-7} \mathrm{~mol} \mathrm{~s}^{-1} \mathrm{~cm}^{-2}, D \approx 10^{-5} \mathrm{~cm}^{2} \mathrm{~s}^{-1}$ and $k_{b} \approx 10^{9} \mathrm{~s}^{-1}$, it would require $k_{S} \ll<2 \times 10^{-7} \mathrm{~cm} \mathrm{~s}^{-1}$. Such a slow electron transfer rate constant is unlikely as photocatalysts are usually large organic molecules with delocalized electronic systems to enable efficient light absorption in the UV-vis domain and hence electron transfer is endowed with a small reorganization energy leading to fast electron transfer. ${ }^{39,40,41}$ This remark is in line with the observation on Figure $2 b$ that the $\mathrm{CV}$ of pyrene under illumination is located at the same potential as in the dark.

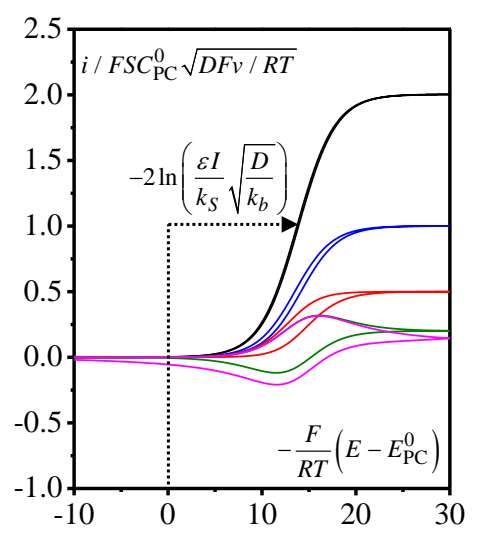

Figure 3. Simulations of CVs in the limiting case $\frac{K \sqrt{\lambda_{b}}}{\Lambda}>>1$ as function of $l=\frac{\delta}{\sqrt{D R T / F v}}=0.5$ (black), 1 (blue), 2 (red), 5 (green), 10 (magenta). $\left(\frac{K \sqrt{\lambda_{b}}}{\Lambda}=10^{3}\right)$

We now analyze the $\mathrm{CV}$ response for the electrophotocatalytic reduction of a substrate $\left(\mathrm{S}_{\mathrm{ox}}\right)$ as part of the e-PC process represented in the left-side of Scheme 3 and recalled in Scheme 7. For the sake of simplicity, we consider a large excess of substrate, i.e. no substrate consumption, and fast catalysis corresponding to pure kinetics conditions on $\mathrm{PC}^{\circ-}$ and implying a steady-state approximation on $\left[{ }^{*} \mathrm{PC}^{\circ}\right]$. As detailed above, we also consider that for systems of interest $K=\frac{\varepsilon I}{k_{b}} \ll 1$. It can thus be shown (see SI) that the CV equation under constant and uniform irradiation is:

$$
\psi=\frac{1}{\frac{\tanh (l \sqrt{\rho})}{\sqrt{\rho}}[1+\exp (-\xi)]+\frac{\exp (-\xi / 2)}{\Lambda}}
$$

The system is thus controlled by three parameters:

$$
\begin{array}{ll}
\Lambda=\frac{k_{S}}{\sqrt{D F v / R T}}, & l=\frac{\delta}{\sqrt{D R T / F v}} \\
\rho & =\frac{k_{c a t} C_{\mathrm{S}}^{0}}{\left(k_{b}+k_{c a t} C_{\mathrm{S}}^{0}\right)} \frac{\varepsilon I}{F v / R T}
\end{array}
$$

and

where $C_{\mathrm{S}}^{0}$ is the substrate concentration and $k_{\text {cat }}$ the bimolecular rate constant for the reaction of $* \mathrm{PC}^{\bullet-}$ with the substrate $S_{o x}$.

Scheme 7. Electrophotocatalytic reduction of a substrate $S_{\mathrm{ox}}$.

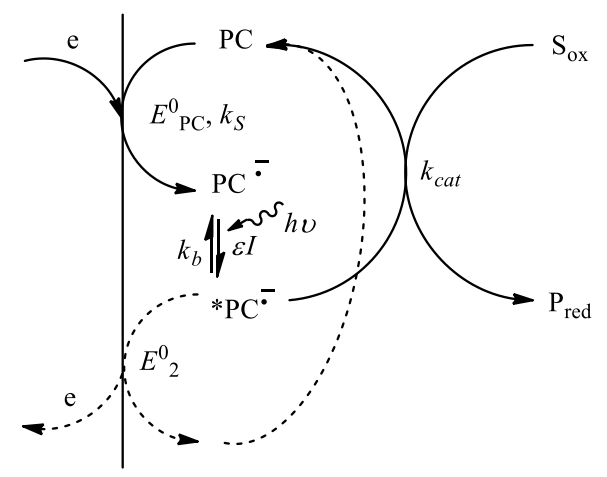

If we take into account that, as already discussed, photocatalyst are large molecules with delocalized orbitals leading to fast electron transfer $(\Lambda \rightarrow \infty)$, we obtain that the electrophotocatalytic $\mathrm{CV}$ is a simple sigmoid with a half-wave potential equal to $E_{\mathrm{PC}}^{0}$ and a plateau current:

$$
i_{p l}=F S C_{\mathrm{PC}}^{0} \sqrt{D} \frac{\sqrt{\frac{\varepsilon I \times k_{c a t} C_{\mathrm{S}}^{0}}{k_{b}+k_{c a t} C_{\mathrm{S}}^{0}}}}{\tanh \left(\frac{\delta}{\sqrt{D / k_{c a t} C_{\mathrm{S}}^{0}}} \sqrt{\frac{\varepsilon I}{k_{b}+k_{c a t} C_{\mathrm{S}}^{0}}}\right)}
$$

Knowing $\varepsilon I, k_{b}$ as well as $\delta$ from the CV study of PC without substrate, the catalytic rate constant $k_{\text {cat }}$ can in principle be extracted from the plateau current. Because the lifetime of $* \mathrm{PC}^{\bullet-}$ is short $\left(k_{b}>10^{9} \mathrm{~s}^{-1}\right)$ it seems reasonable to assume that in most cases $k_{b} \gg k_{c a t} C_{\mathrm{S}}^{0}$, so that the photochemical activation of $\mathrm{PC}^{\bullet-}$ to the strong oxidant $* \mathrm{PC}^{\bullet-}$ is a pre-equilibrium. The apparent pseudo-first order catalytic rate constant is thus $K k_{c a t} C_{\mathrm{S}}^{0}$ leading to a catalytic plateau current:

$$
i_{p l}=F S C_{\mathrm{PC}}^{0} \sqrt{D} \frac{\sqrt{K k_{c a t} C_{\mathrm{S}}^{0}}}{\tanh \left(\frac{\delta}{\sqrt{D / K k_{c a t} C_{\mathrm{S}}^{0}}}\right)}
$$

$\sqrt{D / K k_{c a t} C_{\mathrm{S}}^{0}}$ is the diffusion-reaction layer where catalysis takes place. However, if this thickness is larger than the maximal diffusion layer thickness $\delta$ imposed by the local heating 
due to irradiation, i.e. $\frac{\delta}{\sqrt{D / K k_{c a t} C_{\mathrm{S}}^{0}}} \ll<1$, then $i_{p l}=F S C_{\mathrm{PC}}^{0} D / \delta$. This indicates that catalysis is not fast enough to allow current enhancement when squeezed in a thin layer of layer thickness $\delta$. Alternatively, if $\frac{\delta}{\sqrt{D / K k_{c a t} C_{\mathrm{S}}^{0}}}>$ 1, the plateau current simplifies to: $i_{p l}=F S C_{\mathrm{PC}}^{0} \sqrt{D} \sqrt{K k_{c a t} C_{\mathrm{S}}^{0}}$ and the catalytic rate constant is easily obtained. We note that an evident limitation of using $\mathrm{CV}$ to investigate e-PC is that the condition $\frac{\delta}{\sqrt{D / K k_{c a t} C_{\mathrm{S}}^{0}}}>>1$ may not be easily fulfilled as $K<<1$ due to the short lifetime of the ion radical excited state. Taking again as an potential example the electrophotoreduction of pyrene, ${ }^{31,32}$ in the presence of $m$-chlorotoluene, a catalytic current is observed (see Figure 4 in reference 31 reproduced here in Figure 4) leading to the evaluation of $K k_{c a t} \approx 5 \times 10^{2} \mathrm{M}^{-1} \mathrm{~s}^{-1}\left(D=10^{-6} \mathrm{~cm}^{2} \mathrm{~s}^{-1}\right.$ and $\delta=6.3 \mu \mathrm{m}$, see above). Reduction of m-chlorotoluene by $* \mathrm{PC}^{\bullet-}$ probably leads to a radical that can be easily reduced at the electrode, hence the plateau current takes into account a stoichiometric factor:

$$
i_{p l}=2 F S C_{\mathrm{PC}}^{0} \sqrt{D} \frac{\sqrt{K k_{c a t} C_{\mathrm{S}}^{0}}}{\tanh \left(\frac{\delta}{\sqrt{D / K k_{c a t} C_{\mathrm{S}}^{0}}}\right)}
$$

Note that some doubt can be cast on the exact concentration of pyrene given in reference 31 for the CVs reproduced in Figures $2 \mathrm{~b}$ and 4 , i.e. respectively 5 and $2 \mathrm{mM}$ as the peak current does not seem to scale with the concentration. However we took those concentrations for our rough evaluations. A complete characterization of the system would need to evaluate $K=\frac{\varepsilon I}{k_{b}}$ as well as additional characterization of the photoelectrolysis products. Nonetheless, this example shows that the analytic framework that we propose is realistic and opens opportunities to investigate e-PC mechanisms, get quantitative kinetic characterization and thus improve efficiency.

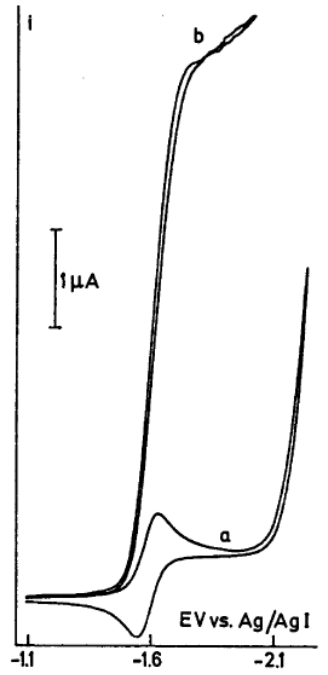

Figure 4. $\mathrm{CVs}$ of pyrene $(2 \mathrm{mM})$ in $\mathrm{DMF}+0.1 \mathrm{M}$ tetrabutylammonium iodide and $m$-chlorotoluene $(0.08 \mathrm{M})$. (a) in the dark, (b) under irradiation $(488 \mathrm{~nm}), v=0.04 \mathrm{~V} \mathrm{~s}^{-1}$. Reproduced from figure 4 in reference 31 .

\section{Conclusion}

We have established the CV responses for electrophotoreduction and electrophotocatalytic systems under realistic conditions and hypothesis. We have shown that cyclic voltammetry is a suitable tool to investigate these processes and get kinetic information. The present analysis reveals, via a dimensionless formulation, that the efficiency of an electrophotocatalytic process depends on the interplay between light absorption, excited state deactivation, reactivity with the substrate and mass transport. In particular, we have shown a catalytic current can only be seen if the size of the diffusion-reaction layer, $\sqrt{D / K k_{c a t} C_{\mathrm{S}}^{0}}$, is smaller than the size of the convection layer induced by local heating of the solution upon irradiation.

The present analysis also provides a useful framework for experimental studies and suggests a step-by-step protocol for the full kinetic characterization of an electrophotocatalytic reaction. Firstly, the $\mathrm{CV}$ analysis of the photocatalyst in the dark and in the absence of substrate allows for evaluation of the interfacial parameters $\left(E_{\mathrm{PC}}^{0}, k_{S}\right.$ and $\alpha$ ) and the diffusion coefficient $(D)$. Secondly, preparation by bulk electrolysis in the dark of the stable ion radical of the photocatalyst $\left(\mathrm{PC}^{\circ-}\right.$ in the case of a reduction) should allow determination of its photophysical properties (extinction coefficient $\varepsilon$ and excited state lifetime $\tau_{b}=1 / k_{b}$ ). This should be the occasion to determine the suitable excitation wavelength where $\mathrm{PC}^{\bullet-}$ absorbs light whereas PC does not. Thirdly, CV analysis of the photocatalyst under illumination (knowing the flux of photon $I$ of the used lamp) and in the absence of substrate should be carried out to evaluate the effect of local heating on convection, i.e. determination of the maximal diffusion layer thickness $\delta(I))$. Finally, $\mathrm{CV}$ analysis under illumination and in the presence of the substrate $S_{\mathrm{ox}}$, allows for an evaluation of the catalytic rate constant $k_{\text {cat }}$.

This approach may be beneficial to the current renewed inter- 
est in electrochemically mediated photoredox catalysis ${ }^{20-26}$ and should allow for quantitative kinetic analysis helping rationalizing the design of such processes.

\section{ACKNOWLEGMENT}

CC thanks Prof. Ally Aukauloo (Université Paris-Saclay) for thoughtful discussions at the initial stage of this work.

\section{ASSOCIATED CONTENT}

\section{Supporting Information}

Derivation of equations. Numerical simulations.

\section{AUTHOR INFORMATION}

\section{Corresponding Authors}

cyrille.costentin@univ-grenoble-alpes.fr

\section{Notes}

The author declares no competing financial interest.

\section{REFERENCES}

(1) Tucker, J. W.; Stephenson, C. R. J. Shining Light on Photoredox Catalysis: Theory and Synthetic Applications. J. Org. Chem. 2012, 77, 1617-1622.

(2) Romero, N. A.; Nicewicz, D. A. Organic Photoredox Catalysis. Chem. Rev. 2016, 116, 10075-10166.

(3) Xi, Y.; Yi, H. ; Lei, A. Synthetic applications of photoredox catalysis with visible light. Org. Biomol. Chem. 2013, 11, 2387-2403

(4) Koike, T. ; Akita, M. Visible-light radical reaction designed by $\mathrm{Ru}$ - and Ir-based photoredox catalysis. Inorg. Chem. Front 2014, 1, 562-576.

(5) Matsui, J. K. ; Lang, S. B. ; Heitz, D. R. ; Molander, G. A. Photoredox-mediated Routes to Radicals : The Value of Catalytic Radical Generation in Synthetic Methods Development. ACS Catal. 2017, 7, 2563-2575.

(6) Stoll, T.; Castillo, C. E.; Kayanuma, M.; Sandroni, M.; Daniel, C.; Odobel, F.; Fortage, J.; Collomb, M.-N. Photo-induced redox catalysis for proton reduction to hydrogen with homogeneous molecular systems using rhodium-based catalysts. Coord. Chem. Rev. 2015, 304-305, 20-37.

(7) Dalle, K. E.; Warnan, J.; Leung, J. J.; Reuillard, B.; Karmel, I. S.; Reisner, E. Electro- and Solar-driven Fuel Synthesis with First Row Transition Metal Complexes. Chem. Rev. 2019, 119, 2752-2875.

(8) Kärkäs, M. D.; Verho, O.; Johnston, E. V.; Åkermark, B. Artificial Photosynthesis: Molecular Systems for Catalytic Water Oxidation. Chem. Rev. 2014, 114, 11863-12001.

(9) Berardi, S. ; Drouet, S. ; Francàs, L.; Gimbert-Suriñach, C.; Guttentag, M.; Richmond, C.; Stoll, T.; Llobet, A. Molecular artificial Photosynthesis. Chen. Soc. Rev. 2014, 43, 7501.

(10) Grätzel, M. Dye-sensitized solar cells. J. Photochem. Photobiol. C. Photochem. Rev. 2003, 4, 145-153.

(11) Zhang, L.; Liardet, L.; Luo, J.; Ren, D. ; Grätzel, M. ; Hu, X. Photoelectrocatalytic arene C-H amination. Nat. Catal. 2019, 2, 366373.

(12) Nocera, D. G. The Artifical Leaf. Acc. Chem. Res. 2012, 45, $767-$ 776.

(13) Breslin, D. T.; Fox, M. A. Excited-State Behavior of Thermally Stable Radical Ions. J. Phys. Chem. 1994, 98, 408-411.

(14) Christensen, J. A.; Phelan, B. T.; Chaudhuri, S.; Acharya, A.; Batista, V. S.; Wasielewski, M. R. Phenothiazine Radical Cation
Excited States as Super-oxidants for Energy-Demanding Reactions. $J$. Am. Chem. Soc. 2018, 140, 5290-5299.

(15) Moutet, J-C.; Reverdy, G. Photochemistry of Cation Radicals in Solution: Photoinduced Electron-transfer Reactions between Alcohols and the $N, N, N^{\prime}, N^{\prime}$-Tetraphenyl-p-phenylenediamine Cation Radical. J. Chem. Soc. Chem. Commun. 1982, 654-655.

(16) Shukla, S. S.; Rusling, J. F. Photoelectrocatalytic Reduction of 4 Chlorobiphenyl Using Anion Radicals and Visible Light. J. Phys. Chem. 1985, 89, 3353-3358.

(17) Robertson, P. K. J.; Eggins, B. R. Photoelectrochemistry with Quinone Radical Anions - Photoassisted Reduction of Halobenzenes and Carbonyl Compounds. J. Chem. Soc Perkin Trans. 2 1994, 18291832 .

(18) MacKenzie, I. A.; Wang, L.; Onuska, N. P. R.; Williams, O. F.; Begam, K.; Moran, A. M.; Dunietz, B. D.; Nicewicz, D. A. Discovery and characterization of an acridine radical photoreductant. Nature 2020, 580, 76-81.

(19) Fujitsuka, M.; Kim, S. S.; Lu, C.; Tojo, S.; Majima, T. Intermolecular and Intramolecular Electron Transfer Processes from Excited Naphthalene Diimide Radical Anions. J. Phys. Chem. B 2015, 119, 7275-7282.

(20) Barham, J. P.; König, B. Synthetic Photoelectrochemistry. Angew. Chem. Int. Ed. 2020, 59, 2-18. DOI: 10.1002/anie.201913767.

(21) Capaldo, L.; Quadri, L. L.; Ravelli, D. Merging Photocatalysis with Electrochemistry: The Dawn of a new Alliance in Organic Synthesis. Angew. Chem. Int. Ed. 2019, 58, 17508-17510.

(22) Huang, H.; Strater, Z. M.; Rauch, M.; Shee, J; Sisto, T. J.; Nuckolls, C.; Lambert, T. H. Electrophotocatalysis with a Trisaminocyclopropenium Radical Dication. Angew. Chem. Int. Ed. 2019, 58, 1331813322.

(23) Zhang, W.; Carpenter, K. L.; Lin, S. Electrochemistry Broadens the Scope of Flavin Photocatalysis: Photoelectrocatalytic Oxidation of Unactivated Alcohols. Angew. Chem. Int. Ed. 2019, 59, 409-417.

(24) Huang, H.; Lambert, T. H. Electrophotocatalytic $S_{N} A r$ Reactions of Unactivated Aryl Fluorides at Ambient Temperature and Without Base. Angew. Chem. Int. Ed. 2019, 59, 658-662.

(25) Cowper, N. G. W.; Chernowsky, C. P.; Williams; O. P.; Wickens, Z. K. Potent Reductants via Electron-Primed Photoredox Catalysis: Unlocking Aryl Chlorides for radical coupling. J. Am. Chem. Soc. 2020, 142, 2093-2099.

(26) Kim, H.; Kim, H.; Lambert, T. H.; Lin, S. Reductive Electrophotocatalysis: Merging Electricity and Light To Achieve Extreme Reduction Potentials. J. Am. Chem. Soc. 2020, 142, 2087-2092.

(27) Peters, B. K.; Rodriguez, K. X.; Reisberg, S. H.; Beil, S. B.; Hickey, D. P.; Kawamata, Y.; Collins, M.; Starr, J.; Chen, L.; Udyavara, S.; Klunder, K.; Gorey, T. J.; Anderson, S. L.; Neurock, M.; Minteer, S. D.; Baran, P. S. Scalable and safe synthetic organic electroreduction inspired by Li-ion battery chemistry. Science $\mathbf{2 0 1 9}$, $363,838-845$.

(28) Savéant, J-M.; Costentin, C. Elements of Molecular and Biomolecular Electrochemistry. $2^{\text {nd }}$ Ed. Wiley, 2019.

(29) Gueret, R.; Poulard L.; Oshinowo, M.; Chauvin, J. ; Dahmane, M. ; Dupeyre, G. ; Lainé, P. P. ; Fortage, J. ; Collomb, M-N. Challenging the $\left[\mathrm{Ru}(\mathrm{bpy})_{3}\right]^{2+}$ photosensitizer with a triazatriangulenium robust organic dye for visible light-driven hydrogen production in water. ACS Catal. 2018, 5, 3792-3802.

(30) Yoshida, J-i.; Shimizu, A.; Hayashi, R. Electrogenerated Cationic Reactive Intermediates: The Pool Method and Further Advances. Chem. Rev. 2018, 118, 4702-4730.

(31) Lund, H.; Carlsson, H. S. Photochemistry of Radical Ions. Acta Chem. Scand. B 1978, 32, 505-509.

(32) Carlsson, H. S.; Lund, H. Photochemistry of Radical Ions. II. Influence of Thermal Convection and Photoexcitation on Voltammetric Curves. Acta Chem. Scand. B 1980, 34, 409-412.

(33) Gumy, J-C.; Vauthey, E. Investigation of the Excited-State Dynamics of Radical Ions in the Condensed Phase Using the Picosecond Transient Grating Technique. J. Phys. Chem. A 1997, 101, 85758580 . 
(34) Gosztola, D.; Niemczyk, M. P.; Svec, W.; Lukas, A. S.; Wasielewski, M. R. Excited Doublet States of Electrochemically Generated Imide and Diimide Radical Anions. J. Phys. Chem. A 2000, 104, 6545-6551.

(35) Fujitsuka, M.; Majima, T. Reaction dynamics of excited radical ions revealed by femtosecond laser flash photolysis. J. Photochem. Photobiol. C 2018, 35, 25-37.

(36) (a) Shida, T. Extinction Coefficient of Azulene Anion Radical and the Yield of Scavengeable Electron in g-Irradiated Organic Glass. J. Phys. Chem. 1969, 73, 4311-4314. (b) Paul, D. E.; Lipkin, D.; Weissman, S. I. Reaction of Sodium Metal with Aromatic Hydrocarbons. J. Am. Chem. Soc. 1956, 78, 116-120.

(37) Gimbert-Surinach, C.; Albero, J.; Stoll, T.; Fortage, J.; Collomb, M-N.; Deronzier, A.; Palomares, E.; Llobet, A. Efficient and Limiting
Reactions in Aqueous Light-Induced Hydrogen Evolution Systems using Molecular Catalysts and Quantum Dots. J. Am. Chem. Soc. 2014, 136, 7655-7661.

(38) see reference 28 chap. 1. p. 53

(39) Kojima, H.; Bard, A. J. Determination of rate constants for the electroreduction of aromatic compounds and their correlation with homogeneous electron transfer rates. J. Am. Chem. Soc. 1975, 97, 6317-6324.

(40) Amatore, C.; Maisonhaute, E.; Simonneau, G. Ohmic drop compensation in cyclic voltammetry at scan rates in the megavolt per second range: access to nanometric diffusion layers via transient electrochemistry. J. Electroanal. Chem. 2000 , 486, 141-155.

(41) see reference 28 chap. 1. pp. 59-63. 